\title{
ARTIFICIAL Eye FOR IN VITRo EXPERIMENTS OF LASER LIGHT INTERACTION WITH Aqueous Media
}

\author{
Clarence P. Cain, ${ }^{\dagger}$ Gary D. Noojin, ${ }^{\dagger}$ Daniel X. Hammer, ${ }^{*}$ Robert J. Thomas, ${ }^{*}$ and \\ Benjamin A. Rockwell ${ }^{*}$ \\ ${ }^{\dagger}$ TASC, 750 East Mulberry, Suite 302, San Antonio, Texas 78212-3159; \\ *Armstrong Laboratory, Optical Radiation Division, 8111 18th Street, Brooks Air Force Base, Texas \\ 78235-5215 \\ (Paper JBO-096 received June 6, 1996; revised manuscript received Oct. 2, 1996; accepted for publication Nov. 1, 1996.)
}

\begin{abstract}
An artificial eye has been designed and assembled that mimics the focusing geometry of the living eye. The artificial eye's focusing characteristics are measured and compared with those of the in vivo system. The artificial eye is used to measure several nonlinear optical phenomena that may have an impact on the laser damage thresholds of the retina produced by ultrashort laser pulses. We chose a focal length of $17 \mathrm{~mm}$ to simulate the rhesus monkey eye, with a visual cone angle of $8.4 \mathrm{deg}$ for a $2.5-\mathrm{mm}$ diameter laser beam input. The measured focal point image diameter was $5.6 \pm 1 \mu \mathrm{m}$, which was 1.5 times the calculated diffractionlimited image diameter. This focusing system had the best $\mathrm{M}^{2}$ of all the systems evaluated. We used the artificial eye to measure the threshold for laser-induced breakdown, stimulated Brillouin scattering, supercontinuum generation, and pulse temporal broadening due to group velocity dispersion. ๑ 1997 Society of PhotoOptical Instrumentation Engineers.
\end{abstract}

Keywords eye; optics; laser; focusing; imaging; breakdown.

\section{INTRODUCTION}

Since the invention of the laser, many investigators have studied applications of lasers in ocular surgery and the determination of safe exposure levels. Even before the advent of lasers, the eye had been an especially interesting area of study because of our dependence on visual function during our lives. Many studies have investigated the physical optical properties of the eye, but these studies were difficult to conduct because of the inaccessibility of sections of the eye in the in vivo system. There is still no definitive answer for the spot size a collimated laser makes on the retina, and the dependence of this spot size on wavelength, refractive error, and aberration.

There are numerous dose-response studies that determined the minimum laser energy required to produce retinal damage as a function of wavelength, ${ }^{1,2}$ pulse width, ${ }^{3-7}$ and repetitive pulses. ${ }^{8,9}$ These studies were performed with various lasers and exposure conditions, yet there were consistent trends in the data that reflected a commonness in the animal models used. Investigations of the interaction between subnanosecond laser pulses and the retina ${ }^{3}$ revealed several phenomena

Address all correspondence to Clarence P. Cain. E-mail: clarence.cain@platinum.brooks.af.mil that did not occur for longer pulses (i.e., nonlinear optical phenomena).

Our laboratory has used various optical setups in an attempt to model the focusing of the eye for in vitro experiments. Originally, a $17-\mathrm{mm}$ focal length aspheric lens was used to emulate the reduced focal length of the rhesus monkey eye. ${ }^{10-13}$ The measured spot size of the lens was similar to the limited number of in vivo measurements of the beam diameter of the eye. ${ }^{14,15}$ Although complete characterization of the pulse by spot size measurements gave accurate results in laser-induced breakdown (LIB) threshold studies, ${ }^{12,13}$ aberrations present in the system limited its use in other experiments where aberrations may have had a more profound effect. For example, quantification of the percentage of pulse energy lost in generation of an oscillating cavitation bubble would be erroneous if energy lost due to aberrations was not taken into account. The spherical aberrations in the optical setup with the 17-mm focal length lens were due mainly to the lens-air-water interface but were also inherent in the lens. ${ }^{11}$ Therefore, we sought to reduce the amount of aberration in our system. We began by embedding the 17-mm aspheric lens in a cuvette to reduce the lens-air-water interface, with little success. We also performed experiments on a 
setup developed by Vogel et al., ${ }^{16}$ which used a commercially available contact lens embedded in a cuvette along with an achromatic lens beam expander. Finally, we designed an artificial eye with a lens specifically designed to focus in water.

These phenomena, such as laser-induced breakdown, supercontinuum generation, and selffocusing, depend critically upon the focusing characteristics of the optical system. For example, LIB depends upon irradiance, and hence, a poorly designed optical system (e.g., an artificial eye designed to focus in air using off-the-shelf optics) will not accurately reflect LIB in the eye. An artificial eye was needed that would accurately reflect the focusing characteristics of an actual eye and provide access for measurements.

The spot size and focal area of the artificial eye must possess the same focusing characteristics as a living eye, such as near-diffraction-limited imaging. ${ }^{17}$ Therefore, we have designed a system with near-diffraction-limited characteristics while focusing in an aqueous solution (e.g., water).

Results from measurements with the artificial eye and in vivo experiments have allowed us to determine the relative contributions of several nonlinear optical phenomena on the minimum visible lesion (MVL) threshold. ${ }^{18}$ The MVL threshold is the energy required to produce a barely perceptible change in the retina that is visible ophthalmoscopically, and is the basis by which laser safety standards are set. In Sec. 2 we discuss the design of the artificial eye; in Sec. 3, the measurement of the beam characteristics produced through the artificial eye; and finally, in Sec. 4, we discuss the data collected in this artificial eye.

\section{DESIGN}

The artificial eye was designed to simulate the optics of the living eye for use in laser damage research. Parameters of reduced schematic eyes for vertebrates were published long ago ${ }^{19}$ that gave typical values and dimensions for the component parts. Hughes ${ }^{19}$ listed man as having a focal length of $22.7 \mathrm{~mm}$ and the macaque monkey as having a $16.1-\mathrm{mm}$ posterior focal point. Polhamus and Cohoon ${ }^{15}$ listed the principal focal length at a reference wavelength of $530 \mathrm{~nm}$ to be $16.8 \mathrm{~mm}$ for the rhesus and $22.4 \mathrm{~mm}$ for the human. The artificial eye was designed to simulate only the geometry of the focal region at the image plane within the retina and did not include the absorptive and reflective properties of the fundus. The structure illustrated in Figure 1 allowed measurement and observation of the laser image, irradiances in the beam, bubble generation, and shock wave expansion, which are critical parameters for evaluation of short-pulse laser interaction with the fundus.

Since our studies for MVL thresholds have used rhesus monkeys for in vivo studies, we chose the focal length for our artificial eye to be $17 \mathrm{~mm}$. The

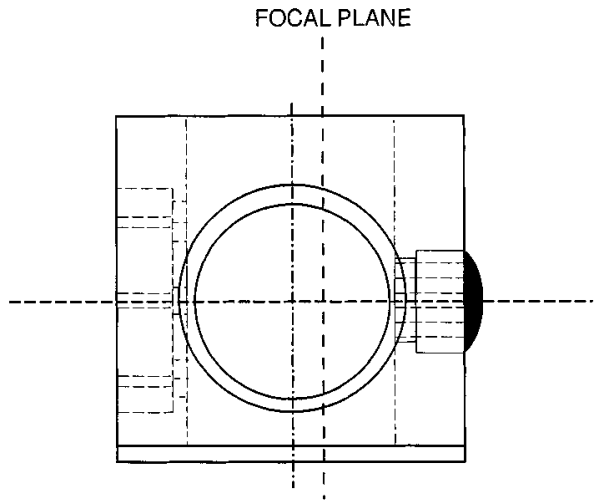

Fig. 1 An engineering drawing of the artificial eye.

lens for the eye was designed to provide a minimal spot size at the focal point within the water or saline, simulating the vitreous humor. For a single wavelength of $580 \mathrm{~nm}$, a single lens design was possible by using a high refractive index glass material and allowing the water to contact the rear surface of the lens. It was necessary to extend the thickness of the glass by $4 \mathrm{~mm}$ and make the back surface plano as shown in Figure 1.

This design provided a near-diffraction-limited spot size within water and the beam waist could be measured with a scanning knife edge. ${ }^{20}$ For most experiments, it was necessary to observe the focal region from two sides and from the rear. For example, continuum generation propagates in the same direction as the laser beam propagation and it is necessary to measure this continuum from the rear of the artificial eye. For LIB and bubble generation threshold measurements, it was necessary to image the bubbles from the side with background lighting. Thus, the artificial eye had to have access from two sides, the rear and the top. Also, it was possible to monitor the artificial eye with a beamsplitter and fundus camera in the same manner as a live eye.

The lens ${ }^{21}$ was designed using a geometrical ray tracing program that allowed each parameter to be varied, including the indices of the component parts. The lens was designed to give a minimal image size for a collimated laser beam input of $2.5-\mathrm{mm}$ diameter at the $580-\mathrm{nm}$ wavelength, and the design gave a $12-\mathrm{mm}$ diameter lens with a $10-\mathrm{mm}$ radius of curvature for the front surface and a plano rear surface. A thickness of $6 \mathrm{~mm}$ was found to be necessary for an index of refraction of $n=1.784$ at $580 \mathrm{~nm}$, that of SF11 glass.

A cube was designed to house the lens as shown in Figure 1 with inside dimensions of 23 - and 8 -mm-thick walls. Quartz windows $25 \mathrm{~mm}$ in diameter were placed on either side and in the back of the cube. The walls were made thick enough to allow the lens and windows to be sealed in the walls with O-rings and screw-held surface rings. With the plano surface of the lens $4 \mathrm{~mm}$ from the inside sur- 
face of the wall, the front surface of the lens protruded past the front wall by $2 \mathrm{~mm}$. Thus, the focal point of the lens was $12 \mathrm{~mm}$ from the plano surface in water and therefore the image was forward 4 $\mathrm{mm}$ from the centerline between the side windows.

\section{Characterization of Performance}

A variety of techniques have been developed in order to characterize the focusing properties of a lens. These typically involve the use of a high-quality laser beam that nearly approximates a Gaussian $\mathrm{TEM}_{00}$ mode. ${ }^{22}$ The beam intensity as a function of position may then be measured near the focal region of a lens. Techniques to make measurements include photographic, fixed aperture, pinhole, moving slit, ronchi ruling, and the knife edge. Among these, the knife-edge measurement offers the most simple implementation for tight-focusing geometries, while providing an excellent assessment of focusing quality. ${ }^{20}$

The knife-edge partially obstructs the beam, depending on its position. This knife edge is assumed to be a perfect intersecting half-plane that is perpendicular to the direction of the beam propagation. This plane is located at some position, $a$, along the beam propagation axis. The total irradiance that propagates beyond the knife-edge is given by the expression:

$$
I(z)=\int_{-\infty}^{\infty} \int_{a}^{\infty} I(x, y, z) \mathrm{d} x \mathrm{~d} y,
$$

when the edge is positioned at $a$ and is obstructing the light from $-\infty$ to $a$. The function that defines $I(x, y, z)$ depends upon the beam properties and the focal geometry. Assuming a collimated Gaussian beam and a focal length $f$ of a single lens, we obtain the following relationship for beam radius $r(z)$ as a function of position $z$ from the minimum radius position $z_{0}, \lambda_{n}\left(\lambda_{n}=\lambda_{0} / n_{0}\right)$ and the diffractionlimited spot size, $r_{0}$ :

$$
r(z)=r_{0}\left\{1+\left[\frac{\lambda_{n}\left(z-z_{0}\right)}{\pi r_{0}^{2}}\right]^{2}\right\}^{1 / 2} .
$$

This is typically expressed in terms of an "Msquared" $\left(\mathrm{M}^{2}\right)$ factor which describes a deviation from a perfect Gaussian diffraction limit. In our case of a short focal length geometry, the commonly accepted modification to the above equation is represented as:

$$
r(z)=M^{2} r_{0}\left\{1+\left[\frac{\lambda_{n}\left(z-z_{0}\right)}{\pi M^{2} r_{0}^{2}}\right]^{2}\right\}^{1 / 2} .
$$

The value of the diffraction-limited beam radius in the focal region of the lens is given by

$$
r_{0(r)}=\frac{\lambda_{n} f}{\pi r_{l}}
$$

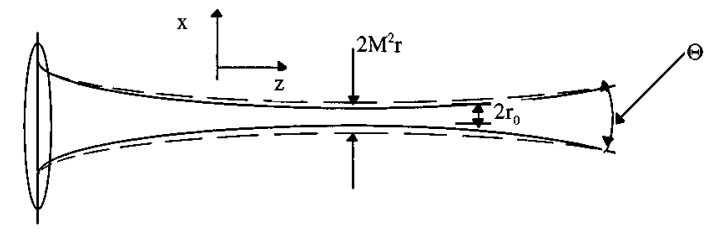

Fig. 2 Schematic to describe the beam characteristics of a nondiffraction-limited beam. The beam is characterized by $M^{2}$ $\left(M^{2} \geqslant 1\right)$. The solid and dashed lines represent a diffraction-limited $\left(M^{2} \equiv 1\right)$ nondiffraction-limited beam $\left(M^{2} \geqslant 1\right)$ propagation.

where $r_{l}$ is the radius of the beam at the principal plane of the lens. The value of $\mathrm{M}^{2}$ gives an important property of the beam in terms of the diffraction-limited beam size. The radius of the focused beam at the minimum waist, $r_{0}$, is given by:

$$
r_{0 \text { (measured) }}=M^{2} r_{0} \text {, }
$$

and the full cone angle, $\theta$, of the focused beam is given by:

$$
\Theta=\frac{2 \lambda_{n}}{\pi r_{0}} .
$$

Figure 2 illustrates these concepts.

The beam radius is defined to be the point at which the irradiance of the beam reaches $1 / e^{2}$ of the maximum. For the knife-edge measurement, the result for a Gaussian beam is that the knife edge cuts the beam at the 16 and $84 \%$ transmission levels along the curve given by the equation:

$$
I(x)=\frac{1}{2}\left[1-\operatorname{erf}\left(\frac{x-x_{0}}{r(z) \sqrt{2}}\right)\right] .
$$

With the above information we have found it possible to accurately determine the geometry of a focused laser beam within the focal region of the artificial eye. An example of the curve fit to the spot size seen is shown in Figure 3 and the results of such measurements are given in Table 1.

\section{DiscUSSION}

\subsection{FOCUSING CHARACTERISTICS}

The setup by Vogel et al. ${ }^{16}$ and the artificial eye both produced exceedingly better results (i.e., a reduction of aberration and hence spot size) than either the 17-mm aspheric lens or the 17-mm aspheric lens embedded in a cuvette. Again, it should be noted that the 17-mm aspheric lens provided excellent results for some experiments as long as the beam profile was fully characterized. Both the Vogel setup and the artificial eye had advantages and disadvantages. The Vogel setup achieved slightly smaller spot sizes and the focusing angle could be adjusted but took a great deal of time to align and was more expensive than the artificial eye; the artificial eye was quick to align and lenses could be easily exchanged in the cuvette but did not focus to 
Knife-Edge Measurement: 100nJ 100fs 580nm

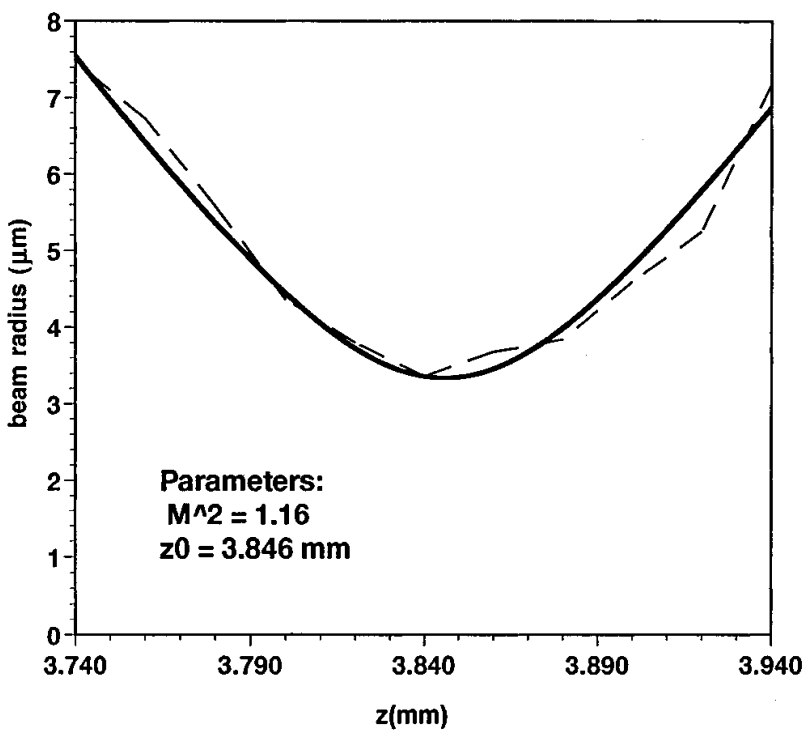

Fig. 3 Knife-edge measurement data for 100-nJ, 100-fs, and 580-nm laser pulses within the artificial eye. The dashed line represents the raw data, and the solid line represents the least-squares fit to Eq. (3).

the spot sizes achieved by the Vogel cell. It should be noted that the original intent of the design of the artificial eye was to emulate the MVL experiments in the rhesus monkey eye. The setup by Vogel et al. was designed to study the effects of laser eye surgery, particularly in the posterior portion of the eye. Thus, the full cone angle of the two setups differed considerably as shown in Table 1 which summarizes the spot size measurements made on each of the four setups.

The artificial eye was used extensively in our laboratory to measure various nonlinear phenomena related to the interaction of laser light with ocular media. In particular, we measured many characteristics of LIB, including breakdown thresholds, ${ }^{18,23}$ generation of shock waves and cavitation bubbles via pump-probe techniques, ${ }^{23}$ time-integrated ${ }^{24}$ and time-resolved ${ }^{25}$ spectroscopic measurements of broadband plasma light, plasma temperature ${ }^{26}$ and the shielding characteristics of breakdown. ${ }^{27}$ Simulated Brillouin scattering results from the interaction of high-intensity laser light with acoustic waves produced upon propagation in water. The SBS threshold was measured for a 10-ns pulse duration. The supercontinuum generation threshold ${ }^{23}$ that results from self-phase modulation (SPM) of high-intensity laser light upon propagation in water was measured at femtosecond and picosecond pulse durations. While SPM produces a broadening of the frequency of the laser light, group velocity dispersion (GVD) produces a temporal broadening of high-irradiation laser light upon interaction with a material that has a suitable linear refractive index gradient, such as water. The following section describes in greater detail each of the nonlinear phenomena measured with the artificial eye.

\subsection{NONLINEAR PHENOMENA MEASURED WITH THE ARTIFICIAL EYE}

\subsubsection{Laser-Induced Breakdown}

LIB is ionization of a significant portion of atoms $\left(10^{18}\right.$ electrons $\left./ \mathrm{cm}^{3}\right)$ by which plasma formation takes place. The resultant plasma can reach very high temperatures $(\sim 10,000 \mathrm{~K})$, and expansion of the plasma creates shock waves and cavitation in liquids. The plasma usually emits broadband visible light (for reported pulse durations greater than or equal to $30 \mathrm{ps}$ ), and this serves as the measured end point for breakdown. For shorter pulse durations, other end points (such as the shock wave or the cavitation bubble) can be used. In our research we have used both the visualization of the residual bubbles and the visualization of the cavitation bubble strobed at $1 \mu$ s after the laser pulse.

Table 1 Comparison of various setups. All measurements were made at a pulse duration of $100 \mathrm{fs}$ and wavelength of $580 \mathrm{~nm}$.

\begin{tabular}{lccccc}
\hline \multicolumn{1}{c}{$\begin{array}{c}\text { Experimental } \\
\text { setup }\end{array}$} & $\begin{array}{c}\text { Input beam } \\
\text { diameter } \\
(\mathrm{mm})\end{array}$ & $\begin{array}{c}\text { Full cone } \\
\text { angle } \\
(\mathrm{deg})\end{array}$ & $\begin{array}{c}\text { Diffraction- } \\
\text { limited } \\
\text { radius } \\
(\mu \mathrm{m})\end{array}$ & $\begin{array}{c}\text { Measured } \\
\text { beam radius } \\
(\mu \mathrm{m})\end{array}$ & $\begin{array}{c}\text { Times } \\
\text { diffraction } \\
\text { limited }\end{array}$ \\
\hline $\begin{array}{l}1.17 \mathrm{~mm} \text { aspheric } \\
\text { 2. Embedded }\end{array}$ & 8 & 27 (calc.) & 0.6 & $8.4 \pm 2.5$ & 14.0 \\
$17 \mathrm{~mm}$ aspheric & 8 & 27 (calc.) & 0.6 & $10.0 \pm 2.0$ & 16.7 \\
3. Vogel setup & $\mathrm{NA}$ & 17.2 (meas.) & 0.9 & $2.2 \pm 0.5$ & 2.4 \\
4. Artificial eye & 2.5 & 8.4 (calc.) & 1.9 & $2.8 \pm 0.5$ & 1.5 \\
\hline
\end{tabular}

a $N A=$ not available. 


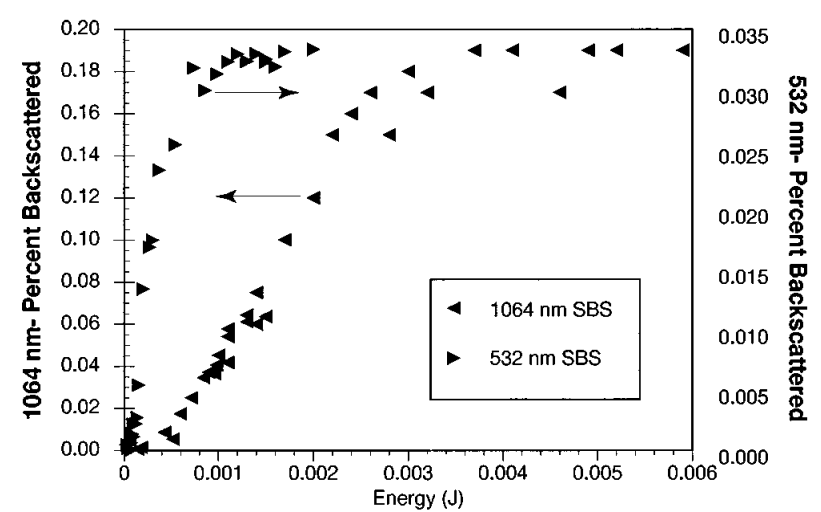

Fig. 4 Stimulated Brillouin scattering as a function of input energy.

LIB is important for both the wide variety of applications that employ breakdown and the potential effect of LIB on ultrashort laser pulse damage of the retina. LIB near the MVL threshold is suspected of occurring in the eye for very short pulses (100 fs) primarily because the MVL threshold and the LIB threshold converge near $100 \mathrm{fs} .{ }^{18}$ At longer pulse durations, the MVL threshold occurs far below the LIB threshold. Recently, interesting histological ${ }^{28}$ and physical $^{18,29}$ evidence has been presented which may lend further credence to the theory of damage mediated by LIB.

The many applications of LIB include ophthalmic microsurgery, laser lithotripsy, laser angioplasty, optical limiting, and laser-induced breakdown spectroscopy (LIBS) for determination of hazardous waste components of materials. The artificial eye has been very well suited for performing measurements on LIB, not only because of its excellent focusing characteristics, but also because the plasma can be viewed directly from many directions simultaneously. This is especially advantageous for timeresolved pump-probe experiments.

\subsubsection{Stimulated Brillouin Scattering}

SBS occurs as a result of electrostriction in various materials, which acts to couple light and acoustic waves. Electrostriction is the tendency of a material to expand or contract in the presence of a large electric field. SBS can occur in the eye because of density fluctuations induced in the vitreous by the large electric fields present in the short pulses produced by an ultrashort pulse laser. Since the response time for SBS is on the order of $1 \mathrm{~ns}$, and the interaction time becomes increasingly shorter as pulse duration decreases, SBS is not observed for picosecond or femtosecond pulses. SBS manifests itself as collimated light scattered in the direction opposite to the propagation direction of the laser light. The threshold for SBS has been measured by Docchio et al. ${ }^{30}$ for an eye model.

Figure 4 shows the percent of laser energy reflected by SBS from the focal region of the artificial eye as a function of input pulse energy at $7 \mathrm{~ns}$, us- ing 1064- and 532-nm light. We find that the percent of energy reflected due to SBS is clamped at $3 \mathrm{~mJ}$ at $1064 \mathrm{~nm}$ and $0.8 \mathrm{~mJ}$ at 532-nm input energy. The quenching of the reflected SBS light represents the maximum amplification of scattered light (by interaction with the input laser light, i.e., the pump beam) for this focusing geometry and material. This does not represent a significant backscatter, and hence we do not expect the MVL threshold to be affected by this small reflected signal.

\subsubsection{Supercontinuum Generation}

Self-phase modulation (SPM) is an effect of the nonlinear refractive index. ${ }^{31,32}$ The effect is not due to the spatial profile of the beam, but rather to the temporal profile of a laser pulse. As the refractive index of a material changes, a time-dependent phase advance or retardation occurs on the time scale of the nonlinear mechanism involved. This phase advance affects the pulse spectral content and can introduce a significant frequency broadening. This broadening, in simple cases of small interaction volumes and fast nonlinearities, can be approximated by the following equation:

$$
\Delta \omega \sim\left(n_{2} \omega_{0} I_{0} L\right) /\left(\tau_{0} c\right),
$$

where $\Delta \omega$ is the maximum extent in frequency that the pulse may be broadened, $I_{0}$ is the peak intensity of the laser pulse, $\tau_{0}$ is the pulse duration, $L$ is the length of the sample through which the propagation occurs, $c$ is the speed of light in a vacuum, and $n_{2}$ is the nonlinear refractive index. One can see that this broadening increases for short pulses and high peak intensities. A broadband emission encompassing the entire visible spectrum may be created from a laser pulse with only a 10-nm bandwidth. SPM is sometimes called "continuum generation" or "superbroadening" because of this dramatic effect. The artificial eye provides an accurate tool that is simple to use, providing experimental access to a well-defined focusing geometry. Table 2 summarizes the thresholds measured for supercontinuum generation in the artificial eye for picosecond to femtosecond pulse durations. Our end point for these measurements was the visualization of a broad spectrum of light through a notch filter with a 20-nm bandwidth and 6 o.d. attenuation at the laser wavelength.

\subsubsection{Temporal Broadening and Group Velocity Dispersion}

In any optical material, the refractive index is dependent upon the frequency of the optical field propagating through the material. This relationship is commonly known as the dispersion function. Owing to this variation of refractive index and the bandwidth of frequency required to produce an ultrashort laser pulse ( $\sim 10 \mathrm{~nm}$ in bandwidth), a pulse can spread simply because the differing wavelengths travel at different speeds. This phenomenon 
Table 2 Summary of measurements made with the artificial eye.

\begin{tabular}{ccccccc}
\hline & & $\begin{array}{c}\text { Breakdown } \\
\text { threshold }(\mu) \\
\text { (Energy trans- } \\
\text { mitted to eye) }\end{array}$ & $\begin{array}{c}\text { Plasma } \\
\text { temperature } \\
(\mathrm{K})\end{array}$ & $\begin{array}{c}\text { Brillouin } \\
\text { scattering } \\
\text { threshold } \\
(\mu)\end{array}$ & $\begin{array}{c}\text { Continuum } \\
\text { generation } \\
\text { threshold } \\
(\mu \mathrm{J})\end{array}$ & $\begin{array}{c}\text { Max-shield- } \\
\text { ing (\% input } \\
\text { energy at 20 } \\
\text { X threshold) }\end{array}$ \\
\hline 7000 & 1064 & $425(151-1190)$ & $6900 \pm 500$ & 700 & - & 88 \\
3000 & 532 & $107(60-190)$ & $6700 \pm 500$ & 110 & - & 93 \\
80 & 1064 & $52(41-65)$ & $7700 \pm 500$ & - & - & 81 \\
60 & 532 & $20(13-31)$ & $7800 \pm 500$ & - & - & 77 \\
3 & 580 & $0.73(0.31-0.72)$ & - & - & 40 & 58 \\
0.3 & 580 & $0.34(0.17-0.71)$ & - & - & 2.0 & 55 \\
0.1 & 580 & $0.27(0.19-0.40)$ & - & - & 0.4 & 73 \\
\hline
\end{tabular}

is referred to as temporal broadening, or group velocity dispersion (GVD). ${ }^{33}$ However, if nonlinear interactions due to $n_{2}$ are considered, GVD can cause complex interactions and irregular pulse shapes. Double or multiple pulses with shifts and splits in the wavelength spectrum can be emitted after transmission through a sample. ${ }^{34}$

Here again we see a unique opportunity to apply the artificial eye in order to characterize an aqueous sample with a well-defined and characterized laser pulse and focusing geometry. Although we have not made a rigorous measurement of the GVD in the artificial eye, preliminary measurements show the temporal broadening to be about 10 to $50 \%$ of the 100 -fs pulse duration for a propagation length of $40 \mathrm{~mm}$ in water.

\subsubsection{Summary of Nonlinear Phenomena Measured}

Table 2 summarizes some of the measurements made with the artificial eye for different pulse durations, where $\tau$ is the pulse duration and $\lambda$ is the wavelength. This table lists the breakdown threshold, the plasma temperature, and the shielding percentage associated with LIB, the SBS threshold, and the supercontinuum generation threshold. The breakdown threshold is defined to be the energy on the probability curve where breakdown occurs $50 \%$ of the time. Also listed in Table 2 are the 10 and $90 \%$ probability values (in parentheses) for comparison. The maximum shielding from LIB is found from the curve fits of the energy transmission data. The SBS thresholds are found by defining threshold to occur for $10 \%$ of the clamped energies shown in Figure 4 . The supercontinuum generation threshold is an approximate value because it was determined in a more subjective manner than the breakdown thresholds.

\subsection{OTHER MEASUREMENTS POSSIBLE WITH THE ARTIFICIAL EYE}

In addition to the above measurements, the artificial eye can be used in other related experiments, where minimization of aberrations is of concern. Raman scattering, tissue absorption and scattering, and spectroscopic and other nonlinear optical experiments can all be performed with little modification to the artificial eye. In fact, the artificial eye is well suited for any measurement of the interaction of laser light with liquid media.

\section{CONCLUSION}

We have designed and fabricated an artificial eye for biophysical experiments that yields focusing characteristics similar to an in vivo eye. We have developed the design specifications from the physical optical characteristics of the rhesus monkey model. The use of this artificial eye has produced biologically relevant threshold measurements that can be used to analyze relevant nonlinear optical phenomena for retinal damage.

\section{ACKNOWLEDGMENTS}

TASC Inc. recognizes support through contract F33615-92-C-0017. This work was supported by the Air Force Office of Scientific Research (2312AA92AL014) and Armstrong Laboratory. This work was performed while R. J. Thomas was an NRC/ Armstrong Laboratory postdoctoral associate.

\section{REFERENCES}

1. D. J. Lund and E. S. Beatrice, "Near infrared laser ocular bioeffects," Health Physics 56(5), 631-636 (1989).

2. A. P. Bruckner, J. M. Schurr, and E. L. Chang, "Biological damage threshold induced by ultrashort 2 nd and 4th harmonic light pulses from a mode-locked Nd:glass laser," U.S. Air Force USAFSAM-TR-80-47 (1980).

3. C. P. Cain, C. A. Toth, C. D. DiCarlo, C. D. Stein, G. D. 
Noojin, D. J. Stolarski, and W. P. Roach, “Visible retinal lesions from ultrashort laser pulses in the primate eye," Invest. Ophthalmol. Vis. Sci. 36(5), 879-888 (1995).

4. A. J. Goldman, W. T. Ham, and H. A. Mueller, "Mechanisms of retinal damage resulting from the exposure of rhesus monkeys to ultrashort laser pulses," Exptl. Eye Res. 21, 457-469 (1975).

5. A. J. Goldman, W. T. Ham, and H. A. Mueller, "Ocular damage thresholds and mechanisms for ultrashort pulses of both visible and infrared laser radiation in the rhesus monkey," Exptl. Eye Res. 24, 45-56 (1977).

6. J. Taboada and W. D. Gibbons, "Retinal tissue damage induced by single ultrashort $1060 \mathrm{~nm}$ laser light pulses," Appl. Opt. 17(18), 2871-2873 (1978).

7. A. P. Bruckner and J. Taboada, "Retinal tissue damage induced by 6 psec 530-nm laser light pulses," Appl. Opt. 21(3), 365-367 (1982).

8. R. W. Ebbers and I. L. Dunsky, "Retinal damage thresholds for multiple-pulse lasers," Aerosp. Med. 44, 317-318 (1973).

9. W. D. Gibbons and D. E. Egbert, "Ocular damage thresholds for repetitive pulsed argon laser exposures," SAM-TR-74-1 (1974).

10. S. A. Boppart, C. A. Toth, W. P. Roach, and B. A. Rockwell, "Shielding effectiveness of femtosecond laser-induced plasmas in ultrapure water," SPIE Proc. 1882, 347-354 (1993).

11. D. X. Hammer, R. J. Thomas, G. D. Noojin, B. A. Rockwell, and A. Vogel, "Ultrashort pulse laser-induced bubble creation thresholds in ocular media," Proc. SPIE 2391, 30-40 (1995).

12. P. K. Kennedy, S. A Boppart, D. X. Hammer, B. A. Rockwell, G. D. Noojin, and W. P. Roach, "A first-order model for the computation of laser-induced breakdown thresholds in ocular and aqueous media: Part II-comparison to experiment," IEEE J. Quant. Electron. 31, 2250-2257 (1995).

13. D. X. Hammer, R. J. Thomas, G. D. Noojin, B. A. Rockwell, P. K. Kennedy, and W. P. Roach, "Experimental investigation of ultrashort pulse laser-induced breakdown thresholds in aqueous media," IEEE J. Quant. Electron. 32, 670-678 (1996).

14. L. D. Forster, "Light intensity measurements with a fiber optic microprobe in the laser irradiated rhesus monkey eye," Diss. the University of Texas at Austin (1978).

15. G. L. Polhamus and D. K. Cohoon, "The spread function in the rhesus," Unpublished report, Armstrong Laboratory, Brooks Air Force Base, Texas (1986).

16. A. Vogel, S. Busch, K. Jungnickel, and R. Birngruber, "Mechanisms of intraocular photodisruption with picosecond and nanosecond pulses," Lasers Surg. Med. 15, 32-43 (1994).

17. F. W. Campbell and R. W. Gubisch, "Optical quality of the human eye," J. Physiol. (Lond.) 186, 558-578 (1966).

18. C. P. Cain, C. D. DiCarlo, B. A. Rockwell, P. K. Kennedy, G. D. Noojin, D. J. Stolarski, D. X. Hammer, C. A. Toth, and W. P. Roach, "Retinal damage and laser-induced breakdown produced by ultrashort-pulse lasers," Graefe's Arch. Clin. Exptl. Ophthalmol. 234 Suppl. 1, S28-37 (1996).
19. A. Hughes, "A useful table of reduced schematic eyes for vertebrates which includes computed longitudinal chromatic aberrations," Vision Res. 19, 1273-1275 (1979).

20. A. E. Siegman, M. W. Sasnett, and T. F. Johnston, "Choice of clip levels for beam width measurements using knife-edge techniques," IEEE J. Quant. Electron. 27, 1098-1104 (1991).

21. Virgo Optics, 6736 Commerce Ave., Port Richie, FL 34668.

22. A. E. Siegman, Lasers, University Science Books, Mill Valley, CA (1986).

23. D. X. Hammer, R. J. Thomas, M. Frenz, E. D. Jansen, G. D. Noojin, S. J. Diggs, J. Noack, A. Vogel, and B. A. Rockwell, "Shock wave and cavitation bubble measurement of ultrashort pulse laser-induced breakdown in water," Proc. SPIE 2681, 437-448 (1996).

24. R. J. Thomas, D. X. Hammer, B. A. Rockwell, G. D. Noojin, D. J. Stolarski, and W. P. Roach, "Spectroscopic measurements of laser induced breakdown in water," J. Opt. Soc. Am. B (submitted).

25. R. J. Thomas, D. X. Hammer, G. D. Noojin, D. J. Stolarski, B. A. Rockwell, and W. P. Roach, "Time-resolved spectroscopy of laser-induced breakdown in water," Proc. SPIE 2681, 402410 (1996).

26. D. J. Stolarski, J. Hardman, C. M. Bramlette, G. D. Noojin, R. J. Thomas, B. A. Rockwell, and W. P. Roach, "Integrated light spectroscopy of laser-induced breakdown in aqueous media," Proc. SPIE 2391, 100-109 (1995).

27. D. X. Hammer, R. J. Thomas, M. Frenz, E. D. Jansen, G. D. Noojin, S. J. Diggs, B. A. Rockwell, and A. J. Welch, "'Shielding properties of laser-induced breakdown in water," Opt. Lett. (submitted).

28. C. A. Toth, D. Narayan, C. Osborne, B. A. Rockwell, C. D. Stein, R. E. Amnotte, C. D. DiCarlo, G. D. Noojin, C. P. Cain, and W. P. Roach, "Histopathology of ultrashort laser pulse retinal damage," Proc. SPIE 2681, 375-381 (1996).

29. C. P. Cain, C. D. DiCarlo, G. D. Noojin, R. E. Amnotte, and W. P. Roach, "In vivo laser-induced bubbles in the primate eye with femtosecond pulses," Proc. SPIE 2681, 382-389 (1996).

30. F. Docchio, L. Dossi, and C. A. Sacchi, "Q-switched $\mathrm{Nd}$ :YAG laser irradiation of the eye and related phenomena: an experimental study. III. Experimental observation of stimulated Brillouin scattering in eye models," Lasers Life Sci. 1, 117-124 (1986).

31. W. Lee Smith, P. Lin, and N. Bloembergen, "Superbroadening in $\mathrm{H}_{2} \mathrm{O}$ and $\mathrm{D}_{2} \mathrm{O}$ by self-focused picosecond pulses from a YAG:Nd laser," Phys. Rev. A 15, 2396-2403 (1977).

32. Q. Z. Wang, P. P. Ho, and R. R. Alfano, "Supercontinuum generation in condensed matter," in The Supercontinuum Laser Source, R. R. Alfano, Ed., pp. 33-90, Springer-Verlag, New York (1989).

33. G. P. Agrawal, "Ultrashort pulse propagation in nonlinear dispersive fibers," in The Supercontinuum Laser Source, R. R. Alfano, Ed., pp. 91-116, Springer-Verlag, New York (1989).

34. G. G. Luther, J. V. Moloney, and A. C. Newell, "Selffocusing threshold in normally dispersive media," Opt. Lett. 19(12), 862-864 (1994). 